\title{
Assessment, Planning, and Execution Considerations for Conjunction Risk Assessment and Mitigation Operations
}

\author{
Ryan C. Frigm ${ }^{1}$ and Joshua A. Levi ${ }^{2}$ \\ a.i. solutions, Inc., Lanham, MD, 20706, USA \\ Dimitrios C. Mantziaras ${ }^{3}$ \\ Honeywell Technology Solutions Inc., Columbia, MD, 21046, USA
}

\begin{abstract}
An operational Conjunction Assessment Risk Analysis (CARA) concept is the real-time process of assessing risk posed by close approaches and reacting to those risks if necessary. The most effective way to completely mitigate conjunction risk is to perform an avoidance maneuver. The NASA Goddard Space Flight Center has implemented a routine CARA process since 2005. Over this period, considerable experience has been gained and many lessons have been learned. This paper identifies and presents these experiences as general concepts in the description of the Conjunction Assessment, Flight Dynamics, and Flight Operations methodologies and processes. These general concepts will be tied together and will be exemplified through a case study of an actual high risk conjunction event for the Aura mission.
\end{abstract}

\section{Introduction}

A COMPLETE Conjunction Assessment Risk Analysis (CARA) operations concept requires a seamless interaction between all concerned parties in reacting to potential close approach events. Moreover, routine CARA is the process of quantifying conjunction risk, planning risk mitigation actions (typically in the form of collision avoidance maneuvers), and execution of those actions. These activities are the respective responsibilities of the NASA Robotic CARA Team, Earth Science Mission Operations (ESMO) Flight Dynamics System (FDS) Team, and ESMO Fight Operations Teams (FOT).

There are many metrics utilized by the CARA Team to quantify the risk posed by a close approach event, such as miss distance and probability of collision. Assessing the quality of the orbit determination (OD) solutions used to perform the close approach predictions is as important as the close approach results themselves; for understanding the OD solution quality permits a qualitative assessment of those risk assessment metrics. As part of a larger operations concept, these risk assessments are only useful if they provide sufficient data to mission stakeholders who, based on their risk tolerance, mission constraints, and knowledge of overall mission performance, can make mitigation decisions based on the risk assessments. Once the collision risk is determined by the CARA Team to warrant risk mitigating action, the FDS team is responsible for planning a risk mitigation, or collision avoidance, maneuver. In this planning process, the FDS team must consider the current orbital position of the spacecraft in relation to ground-track control, science requirements, and other orbit constraints. The team also has to consider past maneuver performance and potentially extrapolate performance characteristics into regimes for which there is no past experience. Once the maneuver planning is completed, the FOT is responsible for executing the planned maneuver. In this process, the FOT must consider the current spacecraft state and health. They must establish that proper support has been scheduled to ensure safe execution of the maneuver. This support includes scheduling proper communication and coverage for tracking, telemetry, and command, as well as ground staffing. The FOT must also inform each Instrument Operation Team (IOT) to ensure the instruments are properly configured. In addition, the FOT must generate, validate, and deliver the spacecraft command loads to execute the maneuver and monitor health and performance throughout the maneuver.

This entire process occurs over the timeframe of a few days to a week. This short timeline offers its own challenges, especially in the form of communication and interaction among the teams. Moreover, the process is not

\footnotetext{
${ }^{1}$ Mission Analyst, Mission Services Division, ryan.frigm@ai-solutions.com, AIAA Member.

${ }^{2}$ Mission Analyst, Mission Services Division, joshua.levi@ai-solutions.com, AIAA Member.

${ }_{3}^{3}$ Aura Flight Systems Manager, Earth Science Mission Operations, Dimitrios.C.Mantziaras@nasa.gov
} 
as linear as suggested, but more of a complex, dynamically evolving, fluid interaction between the necessary parties. This paper identifies and addresses these considerations. These experiences will be presented as general concepts in the description of the CARA, Flight Dynamics, and Flight Operations methodologies and processes in Section II. The team interaction will also be developed and shown through a case study of an actual high risk conjunction event for the Earth Observing System (EOS) Aura mission in Section III. Finally, lessons learned resulting from this case study will be presented, and the impact to future CA operations will be described in Section IV.

\section{Nominal Aura Mission \& Process Overview}

\section{A. Mission Overview}

The EOS Aura mission flies in a larger formation of satellites called the Earth Science Constellations, specifically the Afternoon Constellation. Currently comprised of 5 Earth-observing satellites that all nominally operate in 705-km mean equatorial height, sun-synchronous polar orbits., the Constellation allows for collaborative science but also imposes constraints on relative location, both for science requirements and mission and constellation safety. The design of the spacecraft itself also imposes some restrictions on maneuver performance both due to the inability to perform orbit-lowering maneuvers and due to the lack of dedicated attitude control thrusters. Aura must rely on four thrusters that provide both orbit change delta- $\mathrm{v}$ and attitude control during propulsive maneuvers by

off-pulsing each thruster after a specific amount of

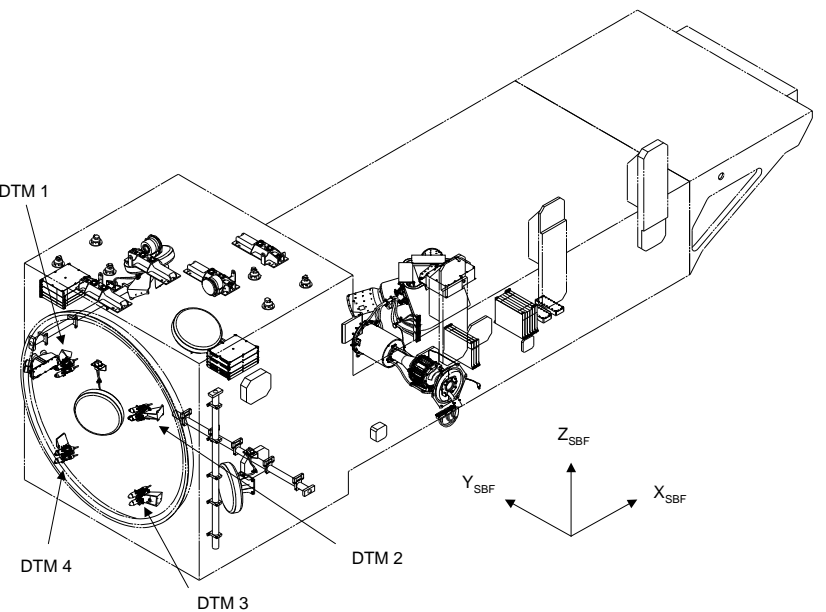

Figure 1: Aura Spacecraft Schematic attitude error is accumulated. This dead band attitude control thrusting prior to and after the delta-v maneuver is somewhat variable on this spacecraft, depending upon the spacecraft initial conditions while entering Attitude Hold Mode (AHM) . This leads to the potential for relatively large delta-v performance errors for the short duration maneuvers, which are typically required to provide conjunction risk mitigation, since attitude control effectively results in additional thrust in the delta-v direction. Modeling of this additional delta-v is based on historical spacecraft performance. Each pair of primary and redundant thruster sets, or dual thruster modules (DTM), as shown in Figure 1, are canted such that the resulting thrust vector extends through the spacecraft center of mass.

It should be noted that Aura's orbit is maintained within a targeted ground track control box of $+/-10$ kilometers centered at +18 kilometers east of Aqua's current Worldwide Reference System-2 (WRS-2) path. This allows Aura to perform the most desirable co-located science with other Afternoon Constellation members. However, the official science requirement is +/- 20 kilometers of allowable ground track error. Table 1 provides an overview of the other mission constraints that must be maintained during the conjunction mitigation process. These requirements are provide for reference, and their impacts will be discussed later in this paper.

Table 1: Aura Orbit Requirements

\begin{tabular}{|l|l|l|}
\multicolumn{2}{|c|}{ Constraint } & \multicolumn{1}{c|}{ Requirement } \\
\hline Ground Track Control & $+/-20 \mathrm{~km}$ WRS-2 (target $+/-10 \mathrm{~km})$ & Mission \& Constellation Safety, Science \\
\hline Eccentricity & $0.0012+/-0.0004$ & Mission \& Constellation Safety, Science \\
\hline Argument of Perigee & $90+/-20$ degrees & Mission \& Constellation Safety, Science \\
\hline Mean Local Time & $13: 30$ to 14:00 & Science \\
\hline
\end{tabular}

\section{B. Conjunction \& Maneuver Assessment Process}

The CARA Team at the NASA/GSFC provides CARA services to NASA's robotic assets, both maneuverable and non-maneuverable. Since 2005, NASA/GSFC has been receiving close approach prediction information from the Joint Space Operations Center (JSpOC), located at Vandenberg Air Force Base (VAFB). The JSpOC "screens" ephemerides of the asset spacecraft against the United States Strategic Command (USSTRATCOM) high accuracy Resident Space Object (RSO) catalog. USSTRATCOM maintains high accuracy element sets for over 19,000 objects in space, including payloads, rocket bodies, and other space debris. The JSpOC screens both its own ephemeris for each asset as well as any Owner/Operator $(\mathrm{O} / \mathrm{O})$-produced ephemeris, when provided, against the catalog. The advantage of screening an $\mathrm{O} / \mathrm{O}$ product is that planned orbit-adjust maneuvers are typically included, 
whereas the JSpOC ephemeris is propagated without knowledge of those future maneuvers. For any identified conjunctions or close approaches between two orbiting space objects, the JSpOC delivers those close approach predictions to GSFC. Using this data, the GSFC CARA Team quantifies the risk associated with the identified conjunctions and provides reports to all mission stakeholders. The CARA Team works closely with mission management, flight dynamics, and flight operations teams to assess the risk and determine what, if any, mitigating actions can be performed to reduce that risk while still achieving the mission constraints.

Pre-processed close approach data is nominally delivered to the CARA Team daily. The initial real-time analysis to calculate Probability of Collision (Pc) and produce trending data is automated and performed as data are received. A CA Summary Report is automatically generated and delivered to all identified and approved mission stakeholders. This report contains information regarding all conjunctions identified with any asset spacecraft. Moreover, it identifies the asset (or primary) spacecraft; secondary object; Time of Closest Approach (TCA); miss distance RSS; radial, along-track, and cross-track separation; conjunction probability; as well as trends of each of these parameters throughout the prediction span. Typically for Low Earth Orbit (LEO) missions, these predictions are made seven days into the future.

As part of the routine CA operations, the CARA Team reviews each conjunction and dispositions the risk or threat level associated with it. This process of dispositioning and determining conjunction risk requires significant CA and mission operations experience. Conjunction risk assessment is the analysis and process of translating the close approach predictions (object states, covariances, and associated orbit determination data) into actionable information for mission stakeholders. Conjunction risk assessment includes assessing the quality of the OD data being used and quantifying and analyzing the risk associated with a predicted conjunction, evaluating risk mitigation options, and determining what actions are necessary. In other words, the CA process is quantifying the risk of the predicted close approach and determining how much confidence should be placed in the quantified risk.

The conjunction geometry, specifically miss distance, is a simple metric that can be easily related to conjunction risk - the smaller the miss distance, the closer the two objects will approach other. The Probability of Collision (Pc) is also a straightforward metric for measuring conjunction risk - the higher the Pc, the more likely that a predicted conjunction will result in a collision. However, these metrics alone do not determine the true conjunction risk. There are many aspects of a conjunction that are analyzed in determining the associated risk, which fall into four general Figures of Merit (FOMs) that factor into the analyst's disposition. These FOMs are conjunction geometry, probability and uncertainty, conjunction evolution or trends, and orbit determination (OD) quality. The first three categories relate to the risk of the close approach. The fourth category, OD quality assessment, evaluates the OD solutions that are used in the close approach prediction process and ultimately determines the validity of the first three FOMs. It also provides the analyst with a measure of the confidence in the covariance generated in the OD process in accurately capturing and reflecting the actual orbit errors of the objects. Without this qualitative assessment, rigorous conjunction assessment could not be performed.

These FOMs and their relation to conjunction risk assessment will become more apparent in the case study presented in Section III. A specific conjunction involving the Aura spacecraft, the integrated approach to analysis of the conjunction, and the subsequent risk mitigation maneuver process will be examined.

\section{Maneuver Planning Process}

The Aura Flight Dynamics team is responsible for maintaining the spacecraft within its designated orbit to assure science objectives are satisfied. This includes planning maneuvers, providing spacecraft attitude support, performing lifetime analyses, and generating products used for spacecraft and instrument operations. During collision avoidance maneuvers, FDS activities begin when the mission management determines that a collision avoidance maneuver may be required based upon the results of the CARA Team analysis. As with a typical orbit maintenance maneuver, these preliminary activities involve determining the time and duration of the maneuver and communicating this information to the FOT to schedule the appropriate ground or space network contacts. Initially, an estimated impulsive delta- $v$ necessary to mitigate the conjunction risk to sufficient level is received from the CARA Team, which will be discussed in the case study.

The Aura spacecraft has the capability to perform two types of propulsive maneuvers: drag make-up (DMU) maneuvers to control its WRS-2 ground track and inclination maneuvers to control its mean local time (MLT) drift. Aura DMU durations are very constrained due to the mission ground track control requirements. To mitigate a conjunction, the Aura FDS team approach is to plan a maneuver similar to a DMU, but typically with a shorter maneuver duration. The maneuver is usually performed $24-48$ hours prior to the predicted TCA. A short-duration burn performed sufficiently prior to TCA will allow the along-track separation component to increase, mitigating the conjunction risk without significantly changing the radial component. For DMU maneuvers, which are intended to increase only the semi-major axis of the orbit, the typical maneuver duration is approximately 6 to 20 seconds 
depending upon the predicted solar activity and atmospheric density. Collision avoidance maneuvers, however, are planned with consideration for the lowest possible maneuver duration required to mitigate the conjunction risk and maintain orbit requirements, while still achieving the equivalent impulsive pro-grade delta-v (Aura does not have the capability to perform retro-grade maneuvers) recommended by the CARA Team.

Selecting an appropriate duration is often the most challenging aspect of collision avoidance maneuvers for the FDS. Since Aura operates in the Afternoon Constellation, maximum maneuver sizes must be evaluated with mission and Constellation safety considerations. Performing a 2-second collision avoidance maneuver may not only violate the goal of maintaining a target WRS-2 error of $+/$ - 10 kilometers, but may threaten to move the spacecraft into the control box of another Afternoon Constellation mission if it moves outside of its required $+/-20$ kilometer ground track error, which is the hard constraint. To avoid this undesirable scenario of close approaches between the Afternoon Constellation members, Aura rarely purposefully flies within the bottom (0 to -10 kilometer) half of its control box. This strategy allows the spacecraft to maintain its typical DMU cycling within 0 to +10 kilometers while keeping 0 to -10 kilometers reserved for collision avoidance maneuvers. Once a collision avoidance maneuver is performed, Aura will have maintained its -10 kilometer WRS-2 science target and will not compromise the safety of the Afternoon Constellation. The minimum burn required is always used to allow capacity for a possible collision avoidance maneuver required before drag has returned Aura to the desired segment of the control box. O/O ephemeris files are produced by FDS for each maneuver duration under consideration and are sent to the JSpOC for screening. The results of those screenings are used to select the best/safest burn duration.

After deciding upon a burn duration that will both mitigate the conjunction and maintain Aura's orbit requirements, maneuver products must be generated and delivered to the FOT. These products are specifically formatted flat ASCII files that contain information pertinent to the maneuver, such as the burn duration, maneuver time, and the commanded attitude. After rigorous quality assurance checks by the FDS team, the files are delivered and again quality assurance checked by FOT personnel, who will use these products to build the command sequences which will be uploaded to the spacecraft. If any part of the maneuver plan needs to be adjusted, either due to updated conjunction information or a change in the maneuver contact, the planning is revisited, resulting in an update to this product set. In addition to the maneuver products, a standard daily set of products are also produced which model the maneuver. These products include specific orbit events, such as day/night transitions and maximum/minimum latitude crossings, which are used by the FOT and instrument teams for planning purposes as well as serve as a notification to other Afternoon Constellation members that a maneuver is currently planned.

Immediately after completion of the maneuver, its performance is assessed by the FDS team via reconstruction and calibration activities. Reconstruction of the maneuver involves using actual on-times to determine each thruster's duty cycle and, assuming the correct thrust scale factor was planned, allows Flight Dynamics to determine the first-order maneuver performance. After an adequate number of Tracking and Data Relay Satellite (TDRS) contacts have occurred allowing an accurate orbit determination to be performed, FDS makes use of a definitive post-maneuver ephemeris to determine the thrust scale factor of the maneuver. This knowledge, coupled with the duty cycles obtained from the reconstruction, allows for a complete assessment of the maneuver's performance. If the change in the spacecraft's semi-major axis was close to the planned change, it is safe to assume that the maneuver was successful in mitigating the conjunction. The post-maneuver orbit is then also used to generate an updated set of daily products produced after the reconstruction and calibration are complete, and will also be screened by the CARA Team on the following day. For assurance that the maneuver was completed nominally, an email notification is delivered to the flight teams of the other Afternoon Constellation member missions.

\section{Maneuver Execution Process}

The primary role of the FOT during the avoidance maneuver process is to execute the maneuver via onboard and real-time commanding, and to monitor the spacecraft performance and health throughout the maneuver to verify that each step of the maneuver occurs successfully. In support of this role, the FOT must work with both the CARA and FDS teams to determine the attributes of the maneuver and generate the operational products needed to perform the maneuver. These products include the maneuver loads in the Stored Command Sequences (SCS), the execution script (Day Plan), Pass Plan Change Requests (PPCRs), and the maneuver Command Authorization Meeting (CAM) package. As the safety of the spacecraft is paramount, the FOT documents in detail the steps for executing important tasks such as planning and executing a maneuver, in the form of Standard Operating Procedures (SOP).

Prior to June 2008, the only established SOP or defined process available to the FOT for planning collision avoidance maneuvers was the nominal Drag Make-Up Maneuver process. This process was established prior to launch based on spacecraft manufacturer, Northrup Grumman, delivered "flows." This process is built on the operational philosophy of separating the three segments of the maneuver (slew out, burn, slew back) and ensuring

4

American Institute of Aeronautics and Astronautics 
that each segment occurs at the precise, predetermined times via onboard SCSs. This makes certain that each portion of the maneuver can be executed independently, and that the execution is not reliant on ground commanding or subject to time variations, in order to provide the maximum safety and predictability. In order to achieve these goals, three SCSs are used (one for each segment) and each SCS contains an absolute time command that triggers the sequence. Using absolute time commands allows for precise timing and predictability, but also greatly limits the flexibility to plan and execute the maneuver.

The process of preparing for and executing an orbit-raising maneuver on Aura contains several steps as shown in Figure 2(a). The steps for a DMU maneuver nominally occur over the course of two weeks to allow time to notify all of the IOT and schedule contacts through the normal Space Network (SN) conflict resolution process .

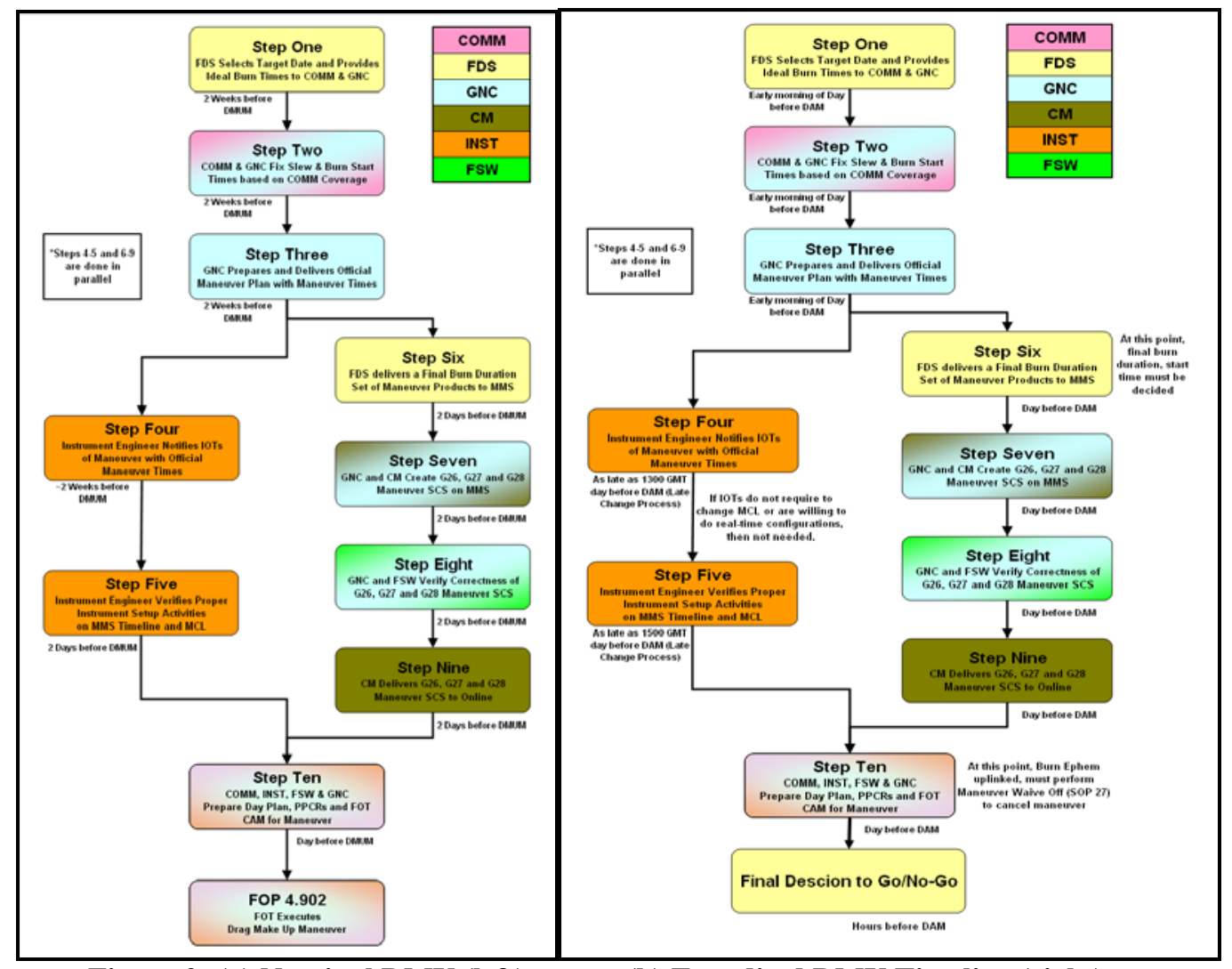

Figure 2: (a) Nominal DMU (left) versus (b) Expedited DMU Timeline (right)

The sequence shown in Figure 2(b) is the same sequence; however the timeline has been compressed to the minimum possible durations given current constraints. Due to the fact that the CA screenings only predict 7 days into the future, the nominal 2 week preparation schedule could not be followed and had to be modified to the schedule on the right. The limiting factors that require preparation to begin the morning of the day before the collision avoidance maneuver are the notification of the maneuver to the IOTs and the building of the SCS (contains instrument reconfigurations if needed as well as communication configuration commands). These times could not be shortened further, because this amount of time is required for the IOTs to reconfigure their instruments if needed and for the nominal merged command load (MCL) generation process to be executed the prior day.

The process flow shown is comprised of 10 steps. The first three steps are executed by Flight Dynamics, Guidance Navigation and Control (GNC), and Communications (COMM) engineers. The FDS engineer provides the target burn date and time, along with the burn duration (step 1). This time is then used by the FOT, GNC, and COMM engineers to correlate the desired maneuver time with the available contact times (step 2). If contact time is not available to support the desired maneuver time, the FOT engineers work with FDS engineers to modify the time to meet the contact visibility requirements for the maneuver. Some key constraints for the maneuver are that the time between slew out end and burn start should be greater than 10 minutes and that the time between burn end and slew back start should be at least 15 minutes. Once the time has been locked down, the GNC engineer will then generate the maneuver plan, which details the timing for each segment of the maneuver, and determine how those 
times match up with orbital night/day, the quaternion values that will be loaded to the spacecraft and when the GNC subsystem onboard will change from Fine-Point Mode to AHM and back (step 3). This plan is then sent out to the IOTs for their planning purposes (step 4). The IOTs may wish, on the basis of this information, to cancel a planned observation or reschedule the timing of an instrument activity via the MCL. Any rescheduled or additional instrument commanding is then verified by the FOT Instrument engineer (step 5). This portion of the flow (steps 4 \& 5) is extremely time critical, as the whole sequence from the time of IOT notification, to updating/rescheduling instrument commanding via the MCL and verification of that MCL must occur within as little as 2 hours. On the Aura mission, all the instrument teams are in different time zones than the FOT, and two of the four are located in Europe. This makes the already tight coordination requirements even more challenging.

With this coordination step complete and the MCL built and verified, the rest of the process can continue. This section (steps 6-9) consists of the final burn parameters being delivered to the FOT's operational planning system in the form of FDS products (step 6). These products are then ingested by the planning system, which provides the information necessary for the FOT GNC engineer to generate the maneuver SCSs (step 7). This is done with configuration management personnel present to insure that loads are properly delivered to the operational system. Once the generation is complete, the GNC engineers generate the SCS reports and verify that every command in each of the three SCSs is correct in both content and timing (step 8). Once the maneuver SCS have been verified, the configuration management (CM) personnel deliver the SCS loads to the real-time command and telemetry system for uplink (step 9).

Following the successful completion of all of the preparation steps, both the FOT and FDS teams generate a slide presentation to be presented at the Command Authorization Meeting (CAM) that explains the details of the maneuver and that all the necessary preparation has been performed (step 10). At the conclusion of the meeting the Mission Director grants approval for the maneuver to proceed. With the approval in hand, all the FOT has left to do is to execute the maneuver.

\section{Assessment, Planning, and Execution Process in Action: An Aura Conjunction Case Study}

In this case study, the coordinated interaction needed to mitigate conjunctions with collision avoidance maneuvers between the CARA, FDS, and FOT teams will become readily apparent through analysis of an actual high interest event. This interaction includes communication and exchange of data and information, and shows the intensity of this exchange during real-time operations as well as the need for a formalized process. The process at the time of this event will be described in this section. Lessons learned and process improvements that helped in the maturation of the CARA process as a result of this event will be described in the next section.

Saturday, 21 June (6 Days Prior to TCA)

On the evening of Saturday, 21 June 2008, a close approach was predicted between Aura and secondary object 01399, a piece of OPS 4682 debris, during a routine conjunction assessment screening. The TCA for this event was Friday, 27 June 2008, approximately 6 days following identification of the close approach event. The Saturday screening, although not typically a nominal screening day, was performed in lieu of a Friday screening due to external considerations. The initial close approach prediction, based on the ASW (Astrodynamics Support Workstation - an acronym for the orbit determination and astrodynamics software used at the JSpOC to produce the close approach predictions) was a total miss distance of 29.8 meters with a corresponding Pc of 7.9E-2. The close approach predictions based on the Owner/Operator $(\mathrm{O} / \mathrm{O})$ ephemeris was 113 meters. The difference between the two solutions observed in the initial prediction is due to differences in modeling and is not unexpected in magnitude.

Sunday, 22 June (5 Days Prior to TCA)

On the morning of Sunday, 22 June, a CA Analyst reviewed the screening results from the previous day. After observing the initial signs of a potential high interest event, e.g. low miss distance and high Pc, the analyst internally notified the CARA Team of the potential event to be worked on Monday morning. Since this event did not appear in a previous screening, and it first entered the screening volume over a weekend when no other routine support was scheduled, there were no communications with the JSpOC or other interested parties until Monday. Nominally, three in-family predictions of an event are desired to show consistency or establish trends in the close approach predictions before any action is recommended. 
Monday, 23 June (4 Days Prior to TCA)

On Monday morning, 23 June, the CARA Team began preliminary detailed analysis of the conjunction. The first analysis performed was conjunction and covariance geometry visualization in which the conjunction angle, defined as the angle between the velocity vectors of the two conjuncting objects, is determined.

The location of the conjunction was predicted to occur at a latitude of 72.9 degrees South and a longitude of 0.4 degrees East. Few sensors had direct observability of the conjunction location. Direct observability would help to reduce OD errors in the predicted state.

The next step was to understand how the covariance and conjunction geometry related to the high Pc computed. To aid in this understanding, an artificial coordinate system can be constructed. For short-encounter conjunctions, the relative position (miss distance) vector is perpendicular to the relative velocity vector. Using this simplification, a right-handed coordinate system can be constructed from the relative position, relative velocity, and cross-product of the two vectors. The conjunction plane is defined by the two-

dimensional plane between the relative position

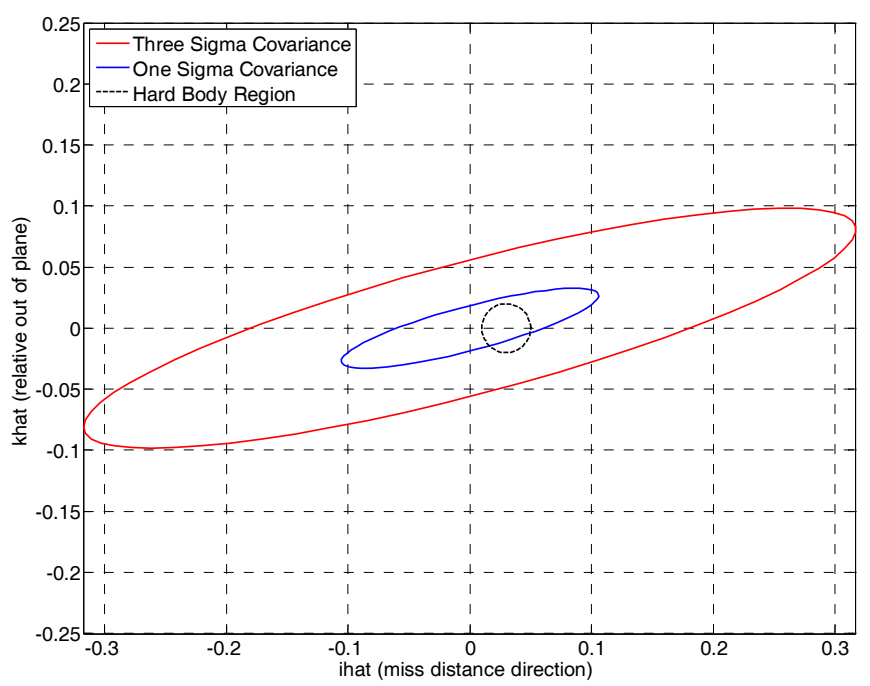

Figure 3: Conjunction Plane Plot and cross-product vectors (i.e. looking down the relative velocity vector). The miss distance is known and can be plotted easily. It is the Hard-Body Radius (HBR), which is an estimate of the combined physical sizes of the objects involved, as a circle of radius HBR at the miss distance direction. By definition, this falls on the $\mathrm{x}$-axis on the conjunction plane plot, off-set from the y-axis by the miss distance. The last step is to project the combined covariance of the two objects onto the conjunction plane. With this plot, as shown in Figure 3, the size, shape, and orientation of the uncertainty can be visualized and measured as it relates to the miss distance and conjunction geometry.

From Figure 3, the clock angle is defined as the angle between the principle axis of the combined error ellipse and the miss distance direction, or the x-axis. For this conjunction, the clock angle was 14.7 degrees. Also from Figure 3 it is observed that the conjunction and covariance geometry is such that a significant component of the combined position uncertainty is in the miss distance direction. The reported Pc is also computed from this conjunction plane. For short-encounter close approaches, the twodimensional Pc can be calculated by integrating this combined covariance probability distribution function (pdf) over the HBR, as shown in Equation 1 [Ref 1]:

$P C=\frac{1}{\sqrt{(2 \pi)^{2}\left|C^{*}\right|}} \iint_{H B R} e^{-\frac{1}{2} \vec{r}^{T} C^{*-1} \vec{r}} d X d Z$

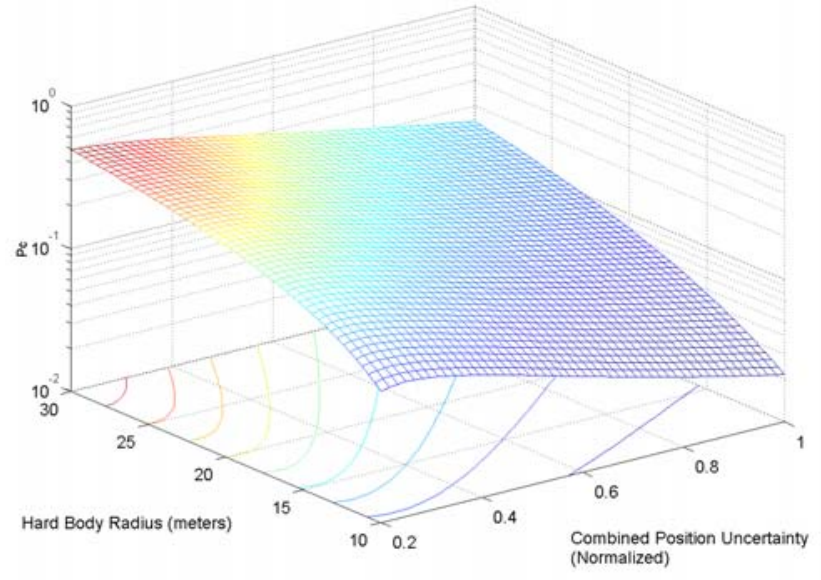

Figure 4: Pc Sensitivity Plot

The final step in the preliminary analysis is to explore the sensitivity of the computed Pc to various input parameters. Figure 4 shows a Pc sensitivity surface plot versus a combined uncertainty scale factor and the HBR used in the Pc calculation. For Pc sensitivity, the scale factor is a scale from 0 to 1, applied to the covariance matrix of both the primary and secondary object. The immediate feature that the CARA team observed was the Pc 
sensitivity to the covariance scale factor. Figure 4 shows that even if the combined covariance, or combined position uncertainty, of the two objects decrease due to increased tracking, the Pc will increase. This initial Pc sensitivity was another indication that this conjunction was of high risk to Aura.

After the preliminary analysis on Monday, which confirmed Sunday's suspicion that this conjunction was of high interest, the CARA Team notified the Aura Mission Director and requested an additional screening update from the JSpOC. It was decided to have an introductory meeting with all the mission stakeholders, including the FDS and FOT, to discuss the conjunction and initial planning. At this point, the CARA Team only had two updates on the conjunction. However, since the CARA Team had already observed early signs of high risk that, from experience, typically leads to avoidance maneuvers, the mission stakeholders began discussing the timeline for decisions.

The CARA Team also presented a maneuver trade space plot. This plot describes the trade-off and various options that exist between maneuver size and phase time (time between maneuver execution and conjunction TCA). The initial maneuver trade space plot is shown in Figure 5, where the contours are lines of constant post-maneuver miss distance (shown in kilometers on Figure 5). The trade space is constructed under the assumption that only velocity or anti-velocity direction maneuvers are performed and that the conjunction is mitigated by allowing the phasing to increase the along-track separation between the two objects. Details of this approach can be found in Reference 2 .

For the Aura spacecraft, only posigrade maneuvers are possible. Therefore, all of the trade space to the left of the zero meters per second delta-v burn is not possible, as indicated

by the shaded red region in Figure 5. From

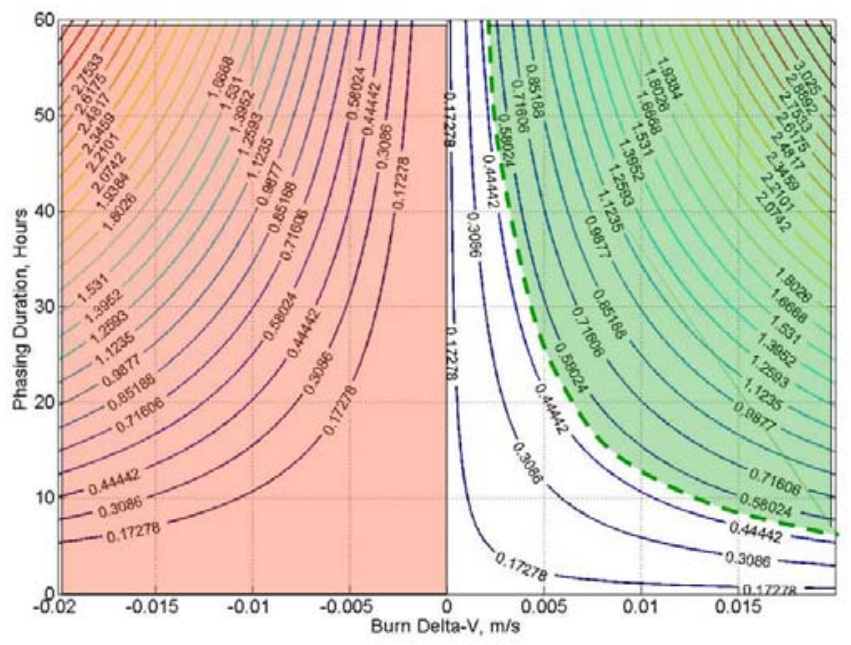
previous analysis, including the conjunction plane plot in Figure 3, the CARA Team determined that a postmaneuver miss distance of at least 300 to 500 meters would be needed to mitigate the conjunction to a sufficient level to drive the Pc towards zero. The required post-maneuver miss distance contour is indicated by the dashed green line in Figure 5. Therefore everything above that line, as indicated by the shaded green region, contains acceptable maneuver trade space options. Due to staffing considerations, it is desirable for maneuvers to be performed during nominal day shifts. In addition, due to the desire to trend the conjunction as much as possible to gather new data, typical maneuver execution times are chosen to be about 24 to 48 hours prior to event TCA.

However, planning this maneuver introduced a unique challenge in that Aura was required to perform the delta- $v$ as noted by the dashed line in Figure 5, which is small in comparison to the typical DMU delta-v. While imparting such a small delta-v on the orbit may cause a negligible effect on the argument of perigee and eccentricity requirements listed in Table 1, it can have a drastic effect on the WRS-2 requirement depending on Aura's position and rate within its control box. In order to maintain Aura's science

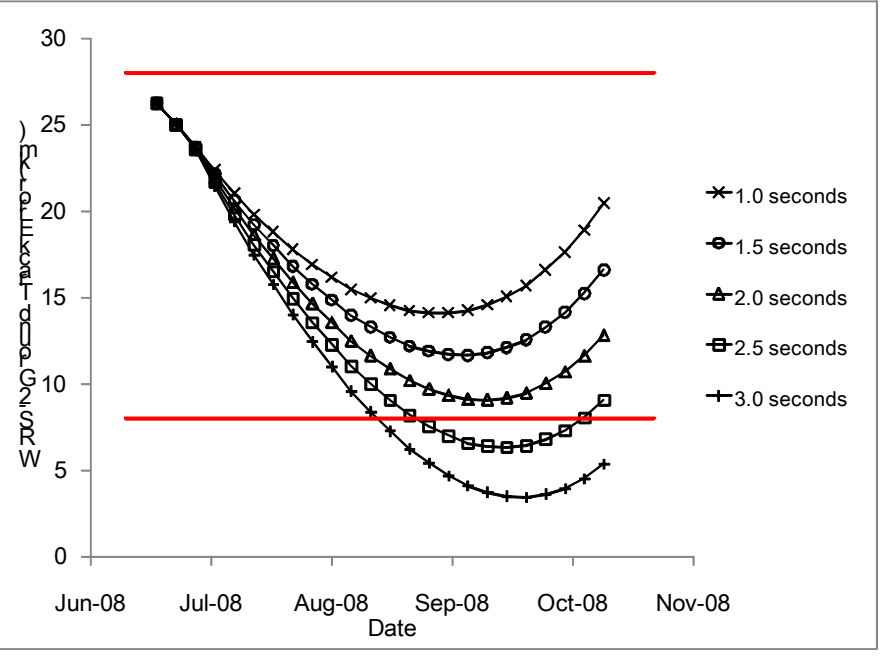

Figure 6: WRS-2 Ground Track Error for Maneuver Options

requirements, the smallest burn duration was used to avoid drifting below the -10 kilometer WRS-2 target. Considering these constraints and historic maneuver performance (Aura's previous shortest maneuver duration was 8.75 seconds), the FDS team selected a 2.0 second burn duration, which was estimated to provide a delta-v of 0.0091 meters per second. Although planning for a larger burn duration would provide greater assurance that the 
conjunction would be mitigated, these larger maneuvers did violate the $+/-10$ kilometer WRS-2 target for Aura, as illustrated by Figure 6 .

The burn duration was then used to determine the ideal time of a one-burn maneuver sequence that would maintain the spacecraft within the frozen orbit requirements of the mission. Since it was unknown when the maneuver would occur relative to the TCA, ideal maneuver times occurring at approximately TCA minus 24 and 48 hours were provided to the FOT to be used as a guideline for scheduling maneuver contacts. Meanwhile, due to the FOT's nominal practice of informing the IOTs and generating the stored command load two days prior to the maneuver, the FOT added additional contacts for Wednesday and developed their maneuver plan should a TCA minus 48 hours DAM be required. As part of this planning process, several discussions were held between the FDS and FOT teams regarding how to properly predict such a short maneuver, since this duration was well outside of the nominal DMU regime. Also, the onboard SCSs were reviewed to ensure that such a short burn duration was feasible with the existing onboard software code. In order to have absolute confidence there would be no issue, a simulation of a 2-second burn was performed on the spacecraft high-fidelity simulator.

Simultaneously with these activities, the CARA Team coordinated with the JSpOC to receive additional updates as well as screen the predicted maneuver ephemeris for any induced conjunctions. The next tag-up occurred in the morning on Tuesday, 24 June. At this point, the CARA Team had received four updates to this event. These close approach update trends were presented to mission stakeholders at the discussion. Additionally, preliminary planning for a Wednesday, 25 June TCA minus 48 hour maneuver had been completed by the FOT and FDS teams.

\section{Tuesday, 24 June (3 Days Prior to TCA)}

All mission stakeholders reconvened again Tuesday, 24 June, in the afternoon. At this point, the CARA Team had received five updates. It was decided by the Mission Director that maneuvering at the TCA minus 48 hour timeframe should be removed from discussion as planning for a TCA-24 hour maneuver allowed for an additional day of conjunction trending before a maneuver decision was needed. This required re-planning of the maneuver, by both the FDS and FOT teams, for the TCA minus 24 hour ideal maneuver time, though it was decided by the FDS team to maintain the same 2.0 second burn duration since this would still provide a large

enough delta-v to allow for an acceptable along-track separation from the object, illustrated in the revisited maneuver trade space shown in Figure 7.

Since retrograde maneuvers are not an option for Aura, that portion of the trade space was removed. The two horizontal black lines provide references for both the TCA minus 24 and TCA minus 48 hour maneuver scenarios. The red dashed line indicates the minimum burn size that Aura could perform without violating its WRS-2 ground track control box. The removal of the TCA minus 48 hour scenario required the FOT to again add additional contacts, rebuild their maneuver plan, and inform the IOTs that a collision avoidance maneuver on Wednesday was no longer being considered and that a Thursday burn had been selected. Another key note to this re-

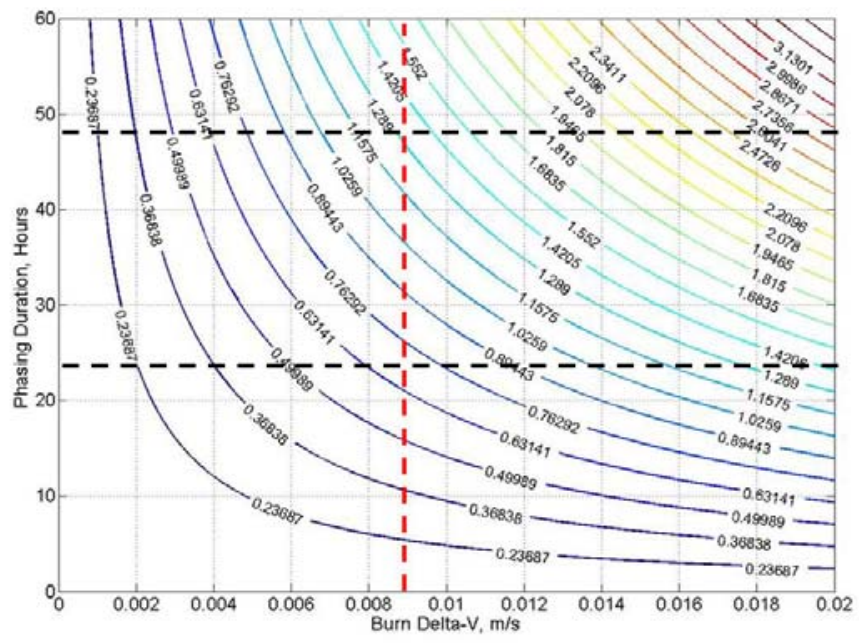

Figure 7: Maneuver Trade Space as of Tuesday PM, 24 June
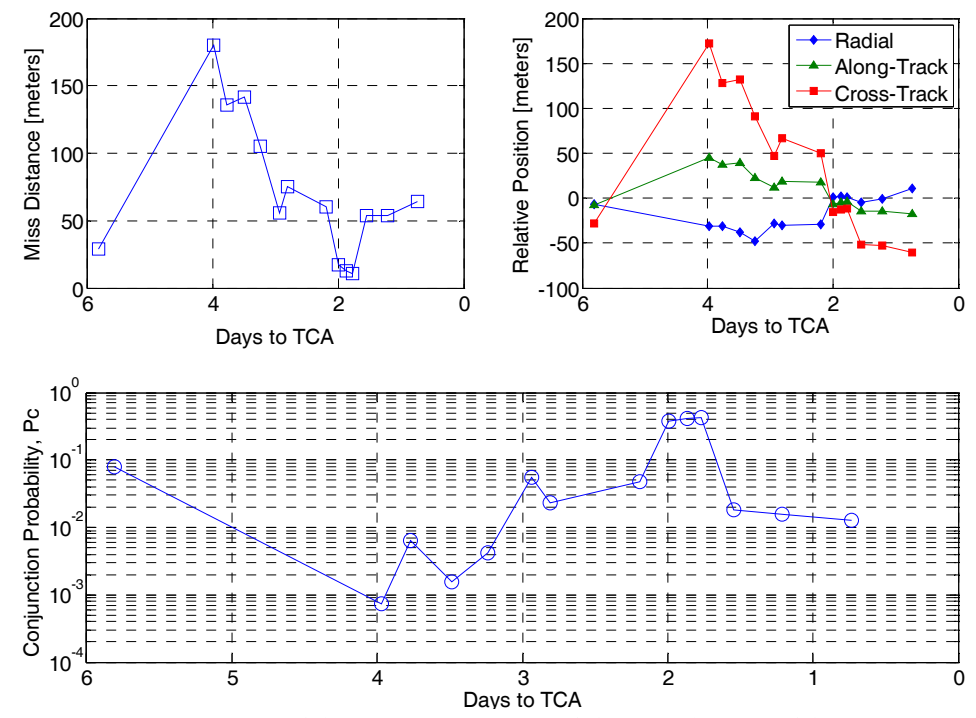

Figure 8: Final (a)Miss Distance, (b) R-I-C, and (c) Pc Trends

9

American Institute of Aeronautics and Astronautics 
planning process is the necessity for the FDS and FOT teams to have quick turnaround and proper communication about the delivery of products for the burn and no burn cases. Should the wrong version of the products get delivered or ingested by the mission planning system, the wrong information would be used to build the stored command load and ephemerides, which could ultimately lead to executing a undesired maneuver without fully knowing implications on the conjunction.

\section{Wednesday, 25 June (2 Days Prior to TCA)}

On Wednesday, 25 June, the teams met one final time for the CAM. The final decision at the CAM is a go/no-go decision on the collision avoidance maneuver. At this point, the CARA Team had six updates, which were presented to mission stakeholders. The FDS team had modeled the nominal maneuver in an ephemeris, and no additional post-maneuver conjunctions were identified when it had been screened by the JSpOC. In addition to the nominal maneuver, the FDS team generated hot and cold maneuver performance cases which were both modeled in ephemerides and screened. Although there were no induced conjunctions by either of the off-nominal maneuver performance cases, the accuracy of these predictions was discussed. At approximately 1 minute before and after the maneuver duration, Aura enters a thruster based attitude hold mode where the spacecraft may require pulses from any of its thrusters (due to initial spacecraft conditions at AHM transition and perturbations during AHM) to maintain its attitude within attitude control system constraints. Considering a burn duration of 2.0 seconds versus the standard DMU maneuver duration of 6 to 20 seconds, the maneuver performance becomes increasingly sensitive to these few, somewhat unpredictable thruster pulses. With so much uncertainty in the predicted maneuver performance, the FDS team bounded the problem by using the extreme hot and cold cases to determine the possible performance envelope. The CARA, FDS, and FOT teams all confirmed ready status to support the maneuver and unanimously recommended executing the nominal planned collision avoidance maneuver on Thursday, 26 June, approximately 24 hours prior to event TCA. Figures 8 a, b, c show the final miss distance; radial, along-track, and cross-track separation; and Pc trends, respectively. From these figures, it is obvious that the initial forecasts from the CARA Team were correct and the event remained of high interest throughout the evolution of the conjunction.

\section{Thursday, 25 June (1 Day Prior to TCA)}

The 2.0 second maneuver was executed successfully by the flight operations team at 14:25:00 GMT. The final predicted postmaneuver miss distance based on the nominal maneuver plan was 652 meters. From post-maneuver reconstruction and calibration, the maneuver performed a delta$\mathrm{v}$ of 0.0122 meters per second resulting in a semi-major axis increase of 23.06 meters versus the nominal plan of 17.36 meters, or 32.9 percent over performance. Although this delta-v was substantially larger than the planned maneuver, it still fell within the advertised predicted performance envelope as indicated in Figure 9. Post-event analysis showed the final close approach miss distance was 795 meters, with a Pc of zero. Assessment, planning, and execution of the collision avoidance maneuver successfully mitigated a potential collision between Aura and a piece of orbiting space debris.

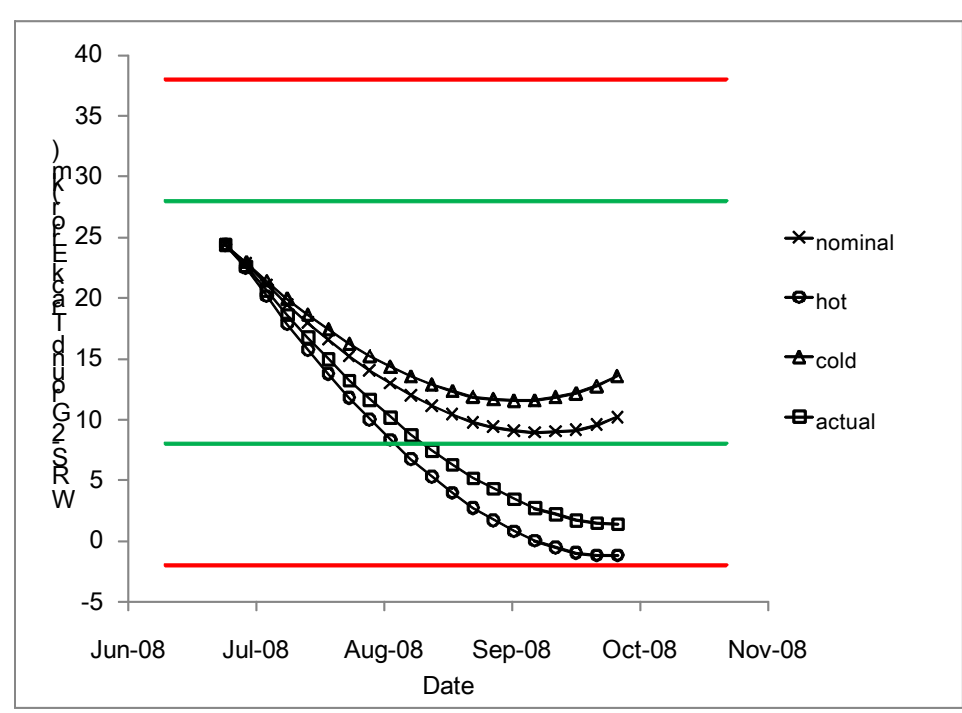

Figure 9: Predicted maneuver performance and actual results with WRS-2 targets and requirements

\section{Maneuver Assessment, Planning, and Execution Lessons Learned \& Process Improvements}

As previously indicated, the process in place at the time of this Aura conjunction was already very mature. However, as a result of this conjunction and subsequent collision avoidance maneuver planning, the CARA, FDS, and FOT Teams learned many valuable lessons and were able to significantly improve their operations even more. This section provides a summary of these lessons learned and process improvements. 


\section{A. Conjunction Assessment}

The miss distance and Pc are two indicators of conjunction risk. However, it is also important to ensure that the input data being used to produce those predictions is of high quality. Simply stated, an effort must be made to ensure that the covariance provided from the OD estimation method accurately reflects the actual orbital errors of the objects. Since the Aura conjunction, one check to ensure that the covariance is bounding the changes in conjunction observed geometry is the miss sigma level plot. The miss sigma level is a comparison of the observed change in relative miss distance components between updates to the previous update's expectation, or, the ability of the covariance to capture expected changes, as shown in Equation 2.

$$
\text { MissSigmaLevel }=\frac{m_{i+1}-m_{i}}{\sigma_{m}}
$$

The miss sigma level can be trended over the evolution of a conjunction. This trend provides a check to ensure the state changes observed in each update is bounded by the statistical expectations from the covariance of the previous update. This addition now provides quantitative assurance which was previously performed qualitatively. Figure 10 provides an example of this trend.

If there is sufficient tracking on the object, then the covariance that results from the OD process should accurately reflect the dynamic errors. Therefore, you would expect the miss sigma level to stay well bounded within 3 sigma range. In the example in Figure 10, the miss sigma level violates the 1-sigma bounds on a few updates and never violates the 3sigma bound; this is representative of a covariance in which the CARA Team would have high confidence. The tighter the miss sigma level is to zero, the better the covariance captures the actual uncertainties.

\section{B. Flight Dynamics}

Aura's first collision avoidance maneuver did successfully mitigate the close approach with the conjuncting object, but the performance of the maneuver relative to the nominal FDS prediction was unsatisfactory. Shortly after this event, an analysis was undertaken to improve the accuracy of future maneuver predictions, specifically maneuvers of a small duration. It was determined that the number of pre- and post-maneuver attitude thruster firings was underestimated in the hot performance case which resulted in a larger change to the semi-major axis than had been planned. In order to rectify the discrepancy, each of Aura's previous maneuvers were analyzed to determine the extent of pre- and post-maneuver attitude thrusting activity and establish a trend to predict the activity of an upcoming maneuver. This would be done by analyzing each of the previous maneuvers in three segments: the premaneuver attitude segment, the actual delta-v, and the post-maneuver attitude segment. This allows for the discrete calculation of duty cycles for each thruster over each segment which can be used to model the thruster activity of a maneuver with a specific duration and known pre- and post-maneuver attitude hold mode durations. This is in contrast to the prior method, where a duty cycle was determined for each thruster based upon the total thrusting that occurred as a function of only the delta-v duration. Several maneuvers were re-planned using the attitude hold mode data from prior maneuvers to determine revised duty cycles, and the results were large improvements in the predicted accuracy of short duration maneuvers. This new method is now currently used to determine the thruster duty cycles of both the Aqua and Aura spacecrafts for short DMU and collision avoidance maneuvers.

For new missions which are held to relatively small ground track requirements, it may be beneficial to plan a few short duration, or small delta-v, maneuvers as part of nominal DMU operations to assess their performance. This would help quantify thruster performance outside of the typical planned DMU durations, and give the mission operators a valuable data point which they can use to plan a short duration maneuver for risk mitigation while maintaining their ground track requirements.

11

American Institute of Aeronautics and Astronautics 


\section{Flight Operations}

Performing the June 2008 close approach allowed the FOT to realize several areas in the current DMU-style maneuver process that were not ideally suited for dealing with a dynamically changing maneuver planning environment like the one leading up to the execution of the June collision avoidance maneuver. This caused the FOT to revisit the current process and identify what changes/enhancements could be made to allow for greater flexibility in planning/executing a collision avoidance maneuver. The three main focuses of these improvements were to eliminate the use of absolute time commands in the maneuver SCSs, allow for a variable burn duration, and determine if the instruments could be configured in real-time versus through the MCL. By eliminating the absolute time commands, the maneuver execution would be based off of ground commanding. This was avoided in the original DMU design due to the fact that it would lead to slight variations in the timing of the maneuver and make the maneuver execution dependent on the communication link. However, when executing a maneuver, the burn durations are typically very small; therefore minor changes (a few seconds) to the start time of the maneuver are insignificant, since the effect on the orbit of the spacecraft is minimal and the evolution of the along-track separation transpires over many hours. With this dependence removed, the FOT is immediately more flexible in terms of response time from when a decision to execute a maneuver is made until when it will be executed.

The second enhancement considered was to remove the fixed burn duration from the maneuver SCSs. This would allow more time for the CARA Team to analyze the conjunction if a maneuver is needed with a very short turnaround. This would allow the FOT to set the burn duration from the ground, independent of the onboard SCSs. Again, allowing for greater flexibility to respond to any DAM scenario.

Also examined was to determine if the yaw slew out and slew back portions ( $\sim+/-14$ degrees for Aura) of the maneuver could be eliminated. Currently, these maneuver segments are executed in order to align the thrust vector with the orbit plane to achieve a pure semi-major axis adjustment. This provides the maximum efficiency in maneuver performance, however when executing a maneuver of only a few seconds, the need to maximize the use of propellant diminishes greatly; the effect on inclination due to not including the yaw offset for a 2-second burn is minimal (you still get 97\% of thrust in the desired direction). With these segments removed, only one SCS would be needed for the entire execution of the maneuver. This would lower the number of required contacts from 3 to 1 and shorten the overall maneuver execution time to just one contact.

Lastly, because the instrument re-configuration is done via the MCL, which must be built the day before the maneuver, the FOT has begun to discuss with the IOTs how the instruments might be configured via real-time commanding. Removing the dependence on the MCL would again shrink the response time as the instrument reconfiguration could be commanded, in real-time, the contact before the execution of the DAM. With all of these enhancements in place, the turn around time could go from 36-48 hours down to only a few hours.

These enhancements are still being analyzed by the FOT and put into place, however for other existing and future flight operations teams these are valuable lessons learned in how to execute a DAM. There certainly exists a trade off between having precise timing and not being reliant on ground commanding when maximizing flexibility in being able to respond to a short turn around conjunction scenario. Given the possibility of a collision with a piece of debris, having a process or sets of processes in place in order to be ready to respond to any scenario, provides the best posture for ensuring the continued safety and successful operations of the spacecraft.

\section{Acknowledgments}

This paper was supported by the NASA/GSFC, under MOMS contract (NNG04DA01C), Task Orders 103 and 209 as well as FDSS contract (NNG10CP02C), Task Orders 12 and 21. Joshua Levi and Ryan Frigm would like to thank their respective Task Monitors, David Tracewell and Lauri K. Newman, for their support of this work. Dimitrios Mantziaras would like to acknowledge and thank John Nidhiry, the lead Aura GNC engineer for his contributions of the maneuver execution timelines. Joshua Levi would also like to thank Eric Palmer, a member of the Aura FDS team, for his efforts in improving the predicted accuracy of maneuver planning. Lastly, the authors would like to thank William (Bill) Guit, Aura Mission Director, for his encouragement of this effort.

\section{References}

${ }^{1}$ Alfriend, K.T., Akella, M.R., Lee, D., Frisbee, J., Foster, J.L., “Probability of Collision Error Analysis,” Space Debris Vol. 1, No. 1, Kluwer Academic Publishers, p. 21-35, 1999. 
${ }^{2}$ McKinley, David P., "Maneuver Planning for Conjunction Risk Mitigation with Ground-Track Control Requirements," 18 $8^{\text {th }}$ AAS/AIAA Space Flight Mechanics Conference, AAS-08-242, Galveston, TX, 2008. 